\title{
Organization of the innervation of the oesophagus and stomach in chinchilla (Chinchilla laniger, Molina)
}

\author{
Elzbieta Nowak
}

Department of Comparative Anatomy, Institute of Biology, Jan Kochanowski University in Kielce, Poland

\begin{abstract}
Using histochemical and histological techniques the morphology and topography of the autonomic plexuses of the oesophagus and stomach in chinchilla (Chinchilla laniger) were investigated. Myenteric and submucosal plexuses were stained for cholinergic and adrenergic structures. Our results showed that the cholinergic structures were formed by networks of various shape and of different size of the neuron agglomerates and nerve fibres. Adrenergic fibres were also found. Density of these structures varied in different parts of the investigated organs. This study is the first attempt to localize the intramural autonomic plexuses in chinchilla. (Folia Histochemica et Cytobiologica 2013, Vol. 51, No. 2, 115-120)
\end{abstract}

Key words: chinchilla, oesophagus, stomach, morphology, autonomic innervation, histochemistry

\section{Introduction}

Studies on the innervation of the digestive tract in vertebrates revealed the presence of intramural plexuses in its wall along the whole length of the alimentary canal [1-5]. Morphological studies on the topography of the myenteric and submucosal plexuses were carried out on different species of amphibians, reptiles, birds and mammals, and revealed significant variability in these structures depending on the systemic position of the animal $[1,3,6-8]$. Chinchilla is a popular species often used as laboratory animal for various studies $[9$, 10]. Nevertheless, most studies concern the pelage, farming conditions, and sense organs, while there is little data on chinchilla's autonomic nervous system. According to the results of comparative anatomy investigations, the level of development of the digestive system is related to the living environment, nutritional needs and metabolism of the investigated species. Also of importance are the differences

\footnotetext{
Correspondence address: E. Nowak,

Department of Comparative Anatomy,

Institute of Biology, Jan Kochanowski University in Kielce;

Swietokrzyska St. 15, 25-406 Kielce, Poland;

e-mail: elzbieta.nowak@ujk.edu.pl
}

in the innervation of the particular segments of the gastrointestinal tract. Because the family of rodents shows wide variation in this aspect, investigations were undertaken into the autonomic innervation of the digestive system in chinchilla. These studies constitute the first part of an analysis of the enteric nervous system in this species.

\section{Material and methods}

The research was conducted on eight adult chinchillas (Chinchilla laniger) of both sexes. All research procedures met the requirements of the $1^{\text {st }}$ Local Ethical Committee in Cracow, certificate No. 19.01.2011. Animals were anesthetized by intraperitoneal administration of Nembutal (20-40 mg/kg). Six animals were used for macromorphological studies and for preparation of frozen sections. Caudal parts of the oesophagus and stomach cardia, fundus and body were dissected, rinsed in physiological saline and fixed for $30 \mathrm{~min}$. in 10\% formalin $(1 \mathrm{~cm}$ segments). The mucosa, submucosa and external muscle layer were separated and whole-mount samples were prepared. Further proceedings were in accordance with the thiocholine method for cholinesterase, and its modification for macromorphological studies and frozen sections [11-13]. For the identification of adrenergic neurons and fibres the sucrose-phosphate-glyoxylic acid 


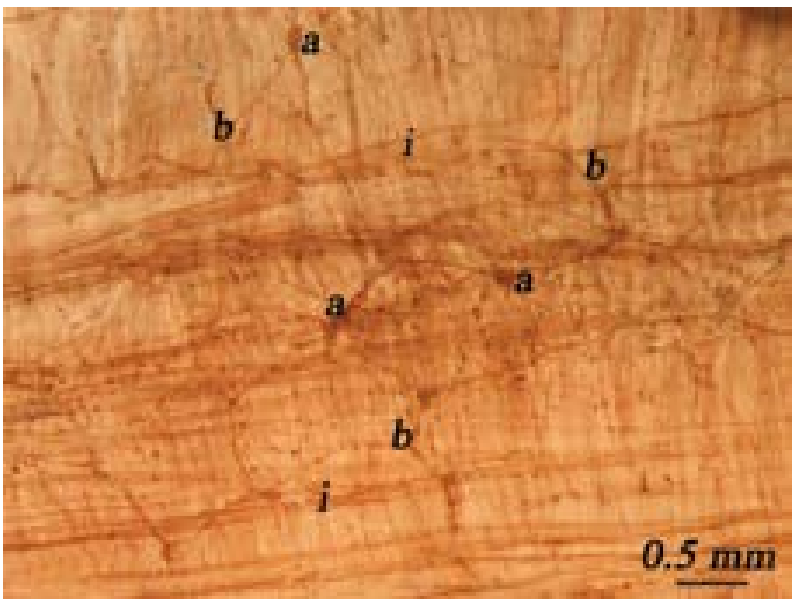

Figure 1. Morphology of the myenteric plexus in the wall of oesophagus - whole-mount specimen (Koelle-Friedenwald method). Abbreviations (this figure and next ones): A autonomic ganglion, $\mathbf{B}$ - cholinergic nerve fibres, $\mathbf{C}$ - first order nerve fibre networks, $\mathbf{D}$ - second order nerve fibre networks, $\mathbf{E}$ - adrenergic nerve fibres, $\mathbf{F}$ - submucosal layer, $\mathbf{G}$ - muscularis layer, $\mathbf{H}$ - mucosa with gastric glands of stomach fundus, I - striated muscles - longitudinal layer, $\mathbf{J}$ - striated muscles - circular layer, $\mathbf{K}$ - external layer smooth muscles, $\mathbf{L}$ - internal layer smooth muscles

(SPG) method was used [14]. Morphometric analyses of oesophagus and stomach sections were performed on 30 fields of approximately $1 \mathrm{~mm}^{2}$ each (from every investigated individual). Moreover, two animals were used for routine histology. Paraffin sections ( 80 slides, $5 \mu \mathrm{m}$ thick) were prepared and stained with the H\&E method. The slides were examined under stereoscopic microscope, Nikon SMZ-800 (Nikon, Tokyo, Japan) and Nikon Eclipse E-400 Epi-Fluorescence Microscope. Measurements (80 cross-sections from each animal) and digital photographs were taken with a Nikon Digital Sight SD-LI system.

\section{Results}

\section{Oesophagus}

The histochemical investigation revealed that the myenteric plexus of the oesophagus was typically located (independent of sex) between the external and internal layers of the striated muscle. The whole mount specimens revealed a scattered network containing a small number of ganglia connected to the bundles of nerve fibres. All these structures were acetylcholinesterase (AChE) positive (Figure 1). The clusters of nerve cells varied in shape: elongated, oval and polygonal. The ganglia were on average (mean \pm SD) $217.6 \pm 113 \mu \mathrm{m}$ long and $125 \pm$ $\pm 77.3 \mu \mathrm{m}$ wide. The thickness of the connecting nerve bundles was on average (mean \pm SD) $18.8 \pm 5.6 \mu \mathrm{m}$.

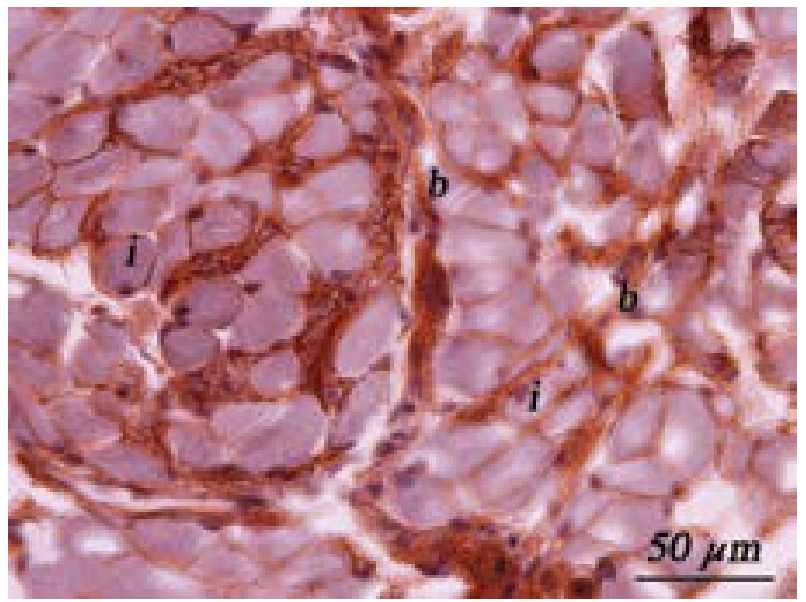

Figure 2. Cholinergic structures of the myenteric plexus in oesophagus - frozen section (Karnovsky-Roots method)

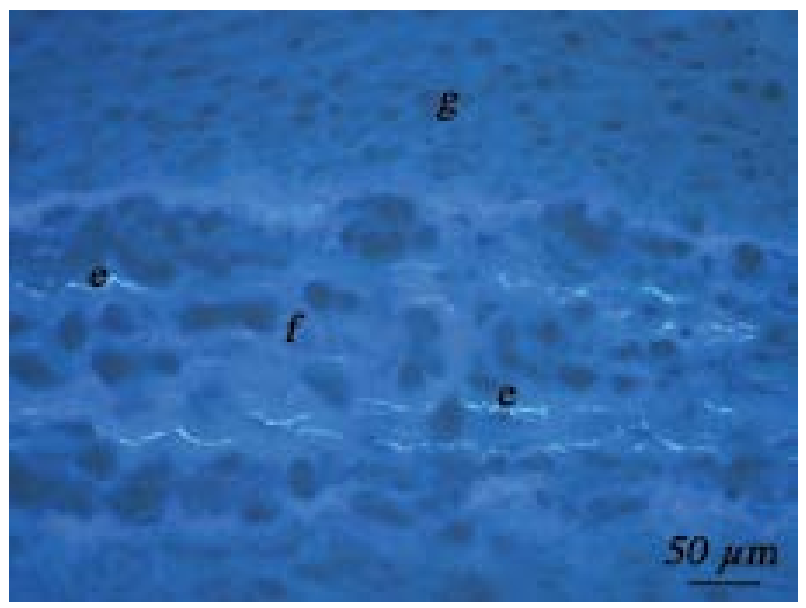

Figure 3. Adrenergic fibres of the submucosal plexus in oesophagus - frozen section (SPG method)

A small number of cholinergic structures was observed in the frozen sections from caudal parts of the oesophagus (Figure 2). These structures located among the striated muscle fibres represented mainly cholinergic fibres which stained in red and brown colour.

The use of the SPG method revealed the presence of adrenergic structures in the myenteric plexus of the oesophagus. They were single varicose nerve fibres located between the internal and external layers of the oesophageal muscles.

The submucosal plexus presented a more dispersed structure and contained a small number of cholinergic fibres and single adrenergic fibres (Figure 3).

Analysis of the routine H\&E stained cross-sections revealed oval or elongated nerve cell aggregations. Ganglia in the caudal part of the oesophagus 


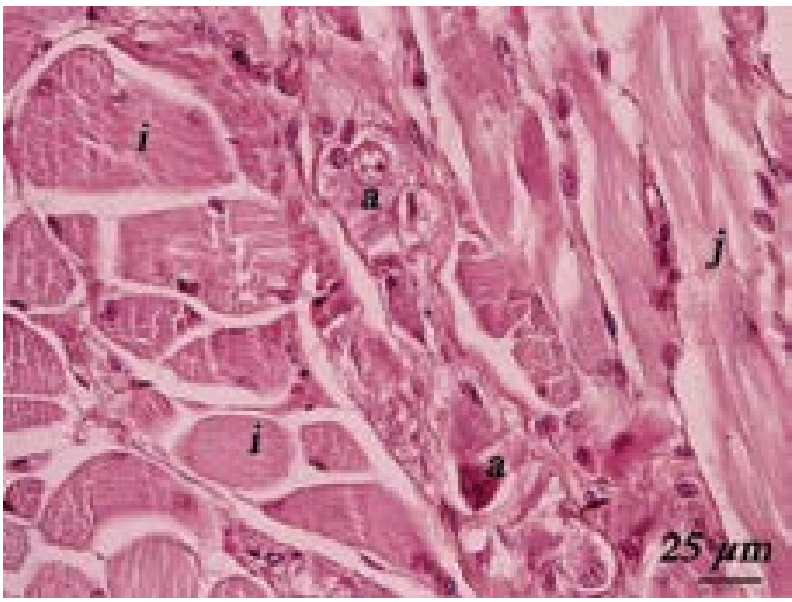

Figure 4. Cross-section through the muscular layer of oesophageal wall (H\&E staining)

were sparse, and were on average (mean \pm SD) $154 \pm$ $\pm 28.6 \mu \mathrm{m}$ long and $22 \pm 8.5 \mu \mathrm{m}$ wide. An average ganglion contained 10-12 neurons. The area of the junction of the caudal oesophageal sphincter with the stomach contained 6-8 ganglia uniformly distributed over the circumference. They were on average (mean \pm SD) $207 \pm 51.7 \mu \mathrm{m}$ long and $31.6 \pm$ $\pm 10.4 \mu \mathrm{m}$ wide (Figure 4).

\section{Stomach}

Histochemical investigations of the stomach's myenteric plexus revealed a distinct and dense cholinergic structure. The plexus was typically distributed (independent of sex) between the internal and external layers of the smooth muscles, and was additionally present within the internal muscular layer. The morphology of the myenteric plexus was different in the various parts of the stomach. The network meshes were of oval shape in the cardiac and pyloric regions and of polygonal shape in the stomach's body. Moreover, intensely stained nerve fibres formed characteristic primary (first-order) thicker and secondary (second-order) thinner networks (Figure 5).

Density analysis of the myenteric plexus revealed about 13 network nodes per $\mathrm{mm}^{2}$. The network meshes were on average (mean \pm SD) $587 \pm 133 \mu \mathrm{m}$ long and $332 \pm 87 \mu \mathrm{m}$ wide, and the thickness of the connecting fibres was on average (mean \pm SD) $21 \pm$ $\pm 7.16 \mu \mathrm{m}$. AChE-positive structures of the Auerbach plexus formed aggregations of ganglionic neurons and nerve fibres between the external and internal layers of muscles. Additionally, single cholinergic fibres ran along myocytes in the inner muscle layer (Figure 6). Moreover, adrenergic fibres visualized by a strong

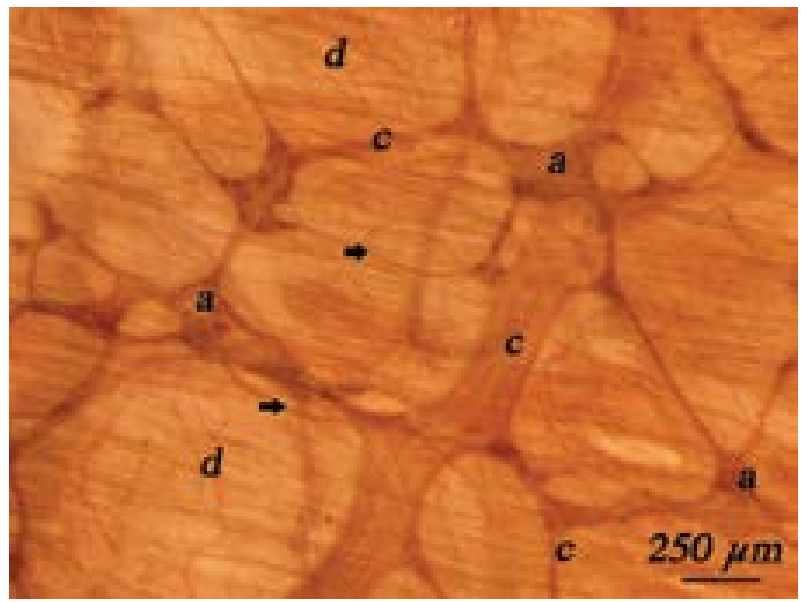

Figure 5. Autonomic nervous system structures in the wall of stomach body (submucosal plexus fibres - arrows) - whole-mount specimen (Koelle-Friedenwald method)

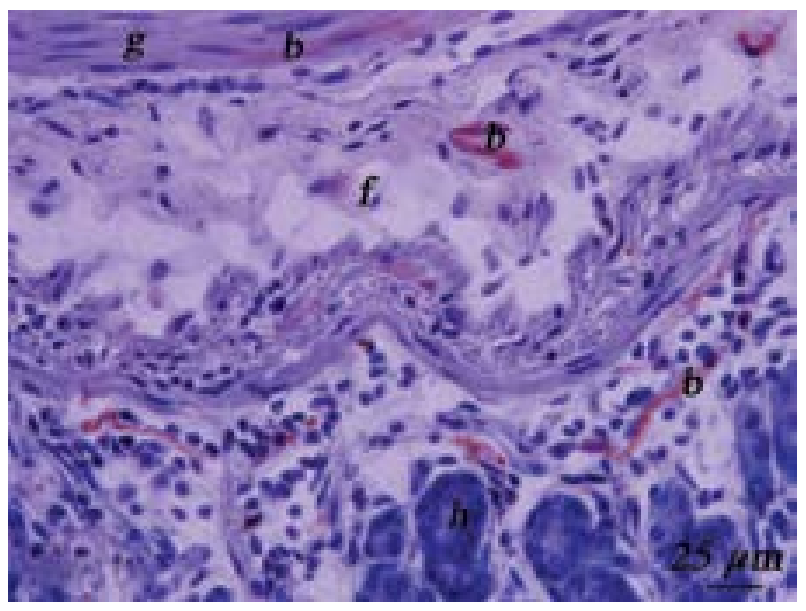

Figure 6. Cholinergic structures in the wall of cardia and body stomach - frozen section (Karnovsky-Roots method)

fluorescence were observed (Figure 7). H\&E staining revealed the elongated shape of nerve cell clusters in the connective tissue between the muscle layers, while oval ganglia were observed in the inner muscle layer. The ganglia usually contained 8-10 neurons and were on average (mean \pm SD) $182 \pm 71.6 \mu \mathrm{m}$ long and $36 \pm$ $\pm 21.5 \mu \mathrm{m}$ wide (Figure 8 ).

The small ganglia of the submucosal plexus were connected by delicate nerve fibres to the ganglia of the Auerbach plexus (Figure 5). They were much smaller in size, on average (mean \pm SD) $140 \pm 31.4 \mu \mathrm{m}$ long and $44 \pm 16 \mu \mathrm{m}$ wide. Intensely stained cholinergic fibres (red colour) were found within the lamina propria of the mucosa in the muscularis mucosae, near the gastric glands (Figure 6). A similar course of the adrenergic fibres was observed (Figure 7). 


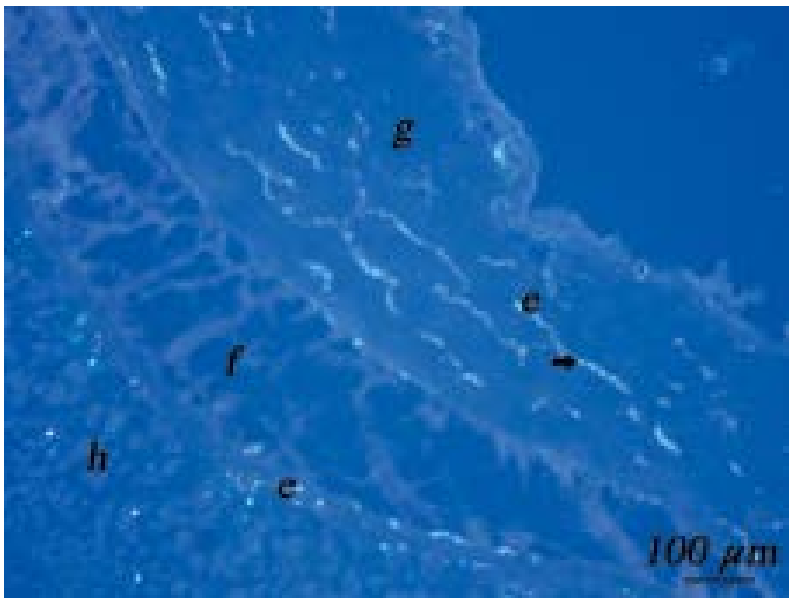

Figure 7. Adrenergic fibres in the wall of stomach body (arrow) - frozen section (SPG method)

\section{Discussion}

The myenteric plexus of the oesophagus in vertebrates has usually been described as a network of autonomic ganglia, diverse in density, connected to nerve bundles. In the analysis of this nervous structure the type of muscle tissue building the wall of the oesophagus has been considered. According to Neuhuber et al. [15] striated muscles were found along the whole length of the oesophagus in rodents, dog and sheep; in other species like cat, opossum, dolphin, macaque and human, the striated muscles of the oesophageal wall were observed at the level of the neck while the thoracic part of the oesophagus contained mainly smooth muscles.

Smooth muscles were found along the whole length of the oesophagus in a lizard (Podarcis hispanica). In this species a well-developed myenteric plexus between the external and internal muscular layers was observed along the entire length of the oesophagus, it revealed high AChE activity [6]. The myenteric plexus as an incompact and irregular plexo-ganglionic network with different expression of AChE activity was observed in the wild mouse (Calomys callosus), hamster, rat and guinea pig. In these species the wall of the oesophagus contained striated muscles [16-19]. A similar level of development of the Auerbach plexus was found in chinchilla.

The wall of the oesophagus in opossum contains both smooth and striated muscles, and different densities of the myenteric plexus were observed in this species. The analysed autonomic plexus was weakly developed between striated muscle layers and significantly better developed in the smooth muscles, particularly in the sphincter of the oeso-

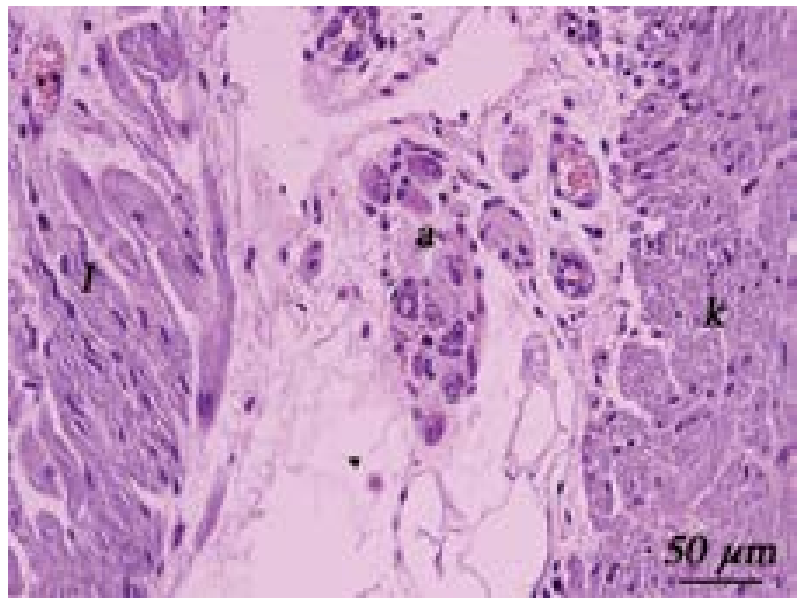

Figure 8. Aggregations of neurons between muscular layers of stomach (H\&E staining)

phagus, where numerous ganglia and nerve fibres were found. Moreover, small aggregations of neurocytes outside the bundles of nerve fibres were observed [7, 20]. Significant scattering of ganglia and an irregular network in the anterior part of the oesophagus were found in guinea pig, while in the cardiac part distinct nodes and bundles of connecting nerve fibres were visible [19]. Similar results were obtained in humans, where 20-35 ganglia per $\mathrm{cm}^{2}$ between the striated muscles in the superior part of the oesophagus were found. The total number of neurons was estimated at between 225000 and 250000 [21].

Analyses of the adrenergic innervation in the myenteric plexus of the oesophagus showed characteristic varicose nerve fibres. These fibres formed a very delicate network, close to the motor plates in hamster [17], while in the lizard (Podarcis hispanica) they formed a network of fibres running parallel to the blood vessels between the layers of the smooth muscles [6]. Similar results were found in chinchilla in this study, with varicose nerve fibres present between the muscular layers.

Studies of the submucosal plexus of the digestive system in vertebrates revealed significant regional differences associated with the functions of specific organs [3]. The macromorphological investigation of the lizard (Podarcis hispanica) oesophagus revealed that this structure is divided into two parts: external and internal. The submucosal external plexus was located below the circular layer of muscles and formed a network with a small number of ganglia and fibres with cholinergic activity [6]. The submucosal internal plexus was better developed and connected to the muscular layer of the mucosa. Autonomic fibres from this part of the submuco- 
sal plexus revealed higher AChE activity than the submucosal external plexus, which penetrated the epithelium. Moreover, the adrenergic fibres came into contact with blood vessels and mucosal glands [6]. Striated muscles build the wall of the oesophagus in wild mouse (Calomys callosus) and hamster, and in these species only nerve fibres formed the submucosal plexus $[17,22]$. In cat, analysis of the density of ganglionic neurons in the submucosal plexus with mixed muscle type revealed the presence of only nerve fibres, and in opossum there was an increase in the number of neurons in the caudal direction [23]. The results obtained in chinchilla in this study are in line with reports in other rodents.

Investigations on the autonomic innervation of the stomach in vertebrates revealed that the system of the intramural plexuses is more similar to the corresponding structures in the intestine than in the oesophagus. Macromorphological investigations of the gastric Auerbach plexus in wild mouse ( $\mathrm{Ca}$ lomys callosus), cat, opossum and rabbit revealed networks with a characteristic shape of meshes, formed by nodes with agglomerations of neurons connected to bundles of fibres $[8,16,20]$.

Analysis of the regional differentiation of the Auerbach plexus of the stomach wall in certain vertebrates - opossum, cat, guinea pig, rabbit and macaque - revealed smaller, narrower and oval network meshes in the region of lesser curvature and much larger and rectangular meshes in the region of greater curvature. Moreover, delicate nerve fibres bypassing the ganglia were observed $[8,20]$. The morphological differentiation of the myenteric plexus in the stomach of chinchilla is generally similar to that of the other mammalian species described here.

Histochemical investigations revealed cholinergic and adrenergic fibres in the myenteric plexus. An AChE-positive network containing first-order (thicker) and second-order fibres with neurons in nodes was observed in wild mouse (Calomys callosus) [16]. Varicosities-forming fluorescent fibres represented the adrenergic structures of this plexus visible both in ganglia and in the connecting bundles. Similar regionally differentiated adrenergic innervation was observed in both muscular layers of the stomach in guinea pig, rat and rabbit. The density of fibres was greatest in the cardiac region and gradually decreased toward the stomach body [24].

The morphology and topography of the submucosal plexus in the stomach of some mammals revealed an incompact network with small ganglia and a majority of thin nerve fibres. A higher density of adrenergic innervation was observed in the caudal part of the oesophagus and cardia in comparison with the fundus and body of the stomach [24]. The adrenergic fibres formed perivascular plexuses and reached the muscularis mucosae and lamina propria, where they branched out around the glands and epithelial cells. Delicate adrenergic fibres in the submucosa and glands of mucosa were also observed in chinchilla.

In conclusion it should be noted that the innervation of the oesophagus and stomach in chinchilla, independent of sex, is generally similar to the structures in rodents, however, the observed differences could be related to the various diet types of the animals.

\section{References}

1. Gun M. Histological and histochemical observations on the myenteric and submucosus plexuses of mammals. J Anat. 1968;102:223-239.

2. Gabella G. Innervation of the gastrointestinal tract. Inter Rev Cytol. 1979;59:129-193.

3. Christensen J. Regional variations in the gross structure of the enteric nervous system: some new views. Trans Am Clin Climatol Assoc. 1990;101:21-37.

4. Hansen MB. The Enteric Nervous System I: Organisation and Classification. Pharmacol Toxicol. 2003;92:105-113.

5. Kleinschmidt S, Nolte I, Hewicker-Trautwein M. Structural and functional components of the feline enteric nervous system. Anat Histol Embryol. 2011;40:450-456.

6. Junquera $C$, Martinez-Ciriano $C$, Castiella $T$ et al. Intrisinic innervation of a reptilian esophagus (Podarcis hispanica). Neurochem Res. 1998;23:493-504.

7. Christensen J, Robinson BA. Anatomy of the myenteric plexus of the opossum esophagus. Gastroenterology. 1982;83:1033-1042.

8. Christensen J, Rick GA. Shunt fascicles in the gastric myenteric plexus in five species. Gastroenterology. 1985;88:1020-1025.

9. Giebink GS. Otitis media: the chinchilla model. Microb Drug Resist. 1999;5:57-72.

10. Hirakawa Y, Sasaki H, Kawamoto E et al. Prevalence and analysis of Pseudomonas aeruginosa in chinchillas. BMC Vet Res 2010;6:52.

11. Karnovsky MJ, Roots L. A direct-coloring thiocholine method for cholinesterases. J Histochem Cytochem. 1964;12:219-221.

12. Koelle GB, Friedenwald JS. A histochemical method for localizing cholinesterase activity. Proc Soc Exp Biol Med. 1949;70:617-622.

13. Gienc J. The application of histochemical method in the anatomical studies on the parasympathetic ganglia and nerve bundles of postganglionic axons in the sublingual region of some mammals. Zool. Pol. 1976;26:187-197.

14. De la Torre JC. An improved approach to histofluorescence using the SPG method for tissue monoamines. J Neurosci Methods. 1980;3:1-5.

15. Neuhuber WL, Raab M, Berthoud HR, J. Wörl J. Innervation of the mammals esophagus. Adv Anat Embryol Cell Biol. SpringerVerlag, Berlin 2006;185:1-76.

16. Maifrino LBM, Prates JC, De-Souza RR, Liberti EA. Morphometry and acetylcholinesterase activity of the myenteric plexus of the wild mouse Calomys callosus. Braz J Med Biol Res. 1997;30:627-632. 
17. Izumi N, Matsuyama H, Yamamoto Y et al. Morphological and morphometrical characteristics of the esophageal intrisinic nervous system in golden hamster. EurJ Morphol. 2002;40:137-144.

18. Greggio FM, Fontes RBV, Maifrino LB et al. Effects of perinatal protein deprivation and recovery on esophageal myenteric plexus. World J Gastroenterol. 2010;16:563-570.

19. Morikawa S, Komuro T. Ultrastructure of intramural ganglia in the striated muscle portions of the guinea pig esophagus. $J$ Anat. 1999;195:111-120.

20. Christensen J, Rick GA, Robinson BA, Stiles MJ, Wix MA. Arrangement of the myenteric plexus throughout the gastrointestinal tract of the opossum. Gastroenterology. 1983;85:890-899.
21. Coimbro DT, Ferraz-de-Carvalho CA, Fujimura I, deSouza RR. Myenteric plexus of the upper part of the human esophagus: A quantitative study. ClinAnat.1994; 7:331-334.

22. Souza NB, Liberti EA, De-Souza RR. Studies on the intrinsic nervous system of the wild rodent Calomys callosus digestive tract. II. The submucosus plexus. Braz J Med Biol Res. 1998;31:647-654.

23. Christensen J, Rick GA. Nerve cell density in submucosus plexus throughout the gut of cat and opossum. Gastroenterology. 1985;89:1064-1069.

24. Costa M, Gabella G. Adrenergic innervation of the alimentary canal. Z Zellforsch. 1971;122:357-377.

Submitted: 8 December, 2012 Accepted after reviews: 24 June, 2013 